\title{
lliopsoas Mass, Hydroureter, and Back Squats: Using Biomechanics and Diagnostic Persistence to Diagnose a Germ Cell Tumor
}

\author{
Andrew C. Berry, DO, ${ }^{1}$ James Fussell, DO, ${ }^{2}$ Gilad Shapira, DO, ${ }^{2}$ Whitney Dawson, $M D,{ }^{2}$ Antwan Hogue, MD $^{2}$ \\ ${ }^{1}$ Division of Gastroenterology, Larkin Community Hospital, South Miami, FL ${ }^{2}$ Department of Medicine, University of South Alabama, \\ Mobile, AL
}

Background: Lesions in the iliopsoas compartment carry a broad differential diagnosis, including infection, tumor, or hematoma, and less commonly, retroperitoneal fibrosis, atrophy secondary to paralysis or muscle disease, foreign body, calcifications secondary to trauma, or rhabdomyolysis. Iliopsoas lesions are oftentimes accompanied by nonspecific symptoms, resulting in a delayed diagnosis.

Case Report: We present the case of a 43-year-old male with acute radiating groin pain who was found to have a traumatic iliopsoas hemorrhagic lesion causing ureteral compression and ureteral dilatation, all presumably originating from a new-onset weight-training program. The patient had a drain placed for fluid removal and decompression, with the initial pathologic specimen confirming muscle fibers and an inflammatory process. Further patient symptomology with combined imaging and pathologic persistence yielded a diagnosis of a germ cell tumor.

Conclusion: The importance of a plausible differential diagnosis combined with clinical diagnostic persistence must be stressed to all clinicians. Our patient's unrelenting inguinal pain led to a justified repeat psoas mass biopsy, imaging, and laboratory workup that led to a diagnosis of psoas germ cell tumor and immediate chemotherapy plan.

Keywords: Compartment syndromes, hematoma, neoplasms-germ cell, psoas muscles

Address correspondence to Andrew C. Berry, DO, Division of Gastroenterology, Larkin Community Hospital, 7000 SW 62nd Ave, Ste 401, South Miami, FL 33143-4717. Tel: (414)617-8877. Email: aberry5555@gmail.com

\section{INTRODUCTION}

Lesions in the iliopsoas compartment carry a broad differential diagnosis and are oftentimes accompanied by nonspecific symptoms, resulting in a delayed diagnosis. ${ }^{1}$ The iliopsoas compartment contains the psoas major, psoas minor, and the iliac muscles-located posterior to the transversalis fascia, the posterior boundary of the retroperitoneum. At first glance, an iliopsoas mass or lesion appears ominous, especially when it causes nearby vessel or organ dislodgement or malfunction. Using appropriate anatomic landmarks and radiographic imaging is imperative to help delineate an accurate diagnosis. The differential diagnosis for iliopsoas lesions is broad and includes infection (primary or secondary), hematomas (spontaneous or secondary), and tumor (primary or secondary). Less commonly, lesions may include retroperitoneal fibrosis, atrophy secondary to paralysis or muscle disease, calcifications secondary to trauma, rhabdomyolysis, or foreign body. ${ }^{2}$ These lesions may compress or dislodge nearby anatomic structures or cause significant organ injury. The diagnostic dilemma necessitates a systematic approach. An initial diagnosis supported by radiographic and histologic evidence may be plausi- ble and accurate; however, a patient with unresponsive or progressive symptoms may warrant a second diagnostic evaluation.

We present the case of a 43-year-old male with acute radiating groin pain who was found to have a traumatic psoas hemorrhagic lesion causing ureteral compression and ureteral dilation. The most plausible etiology was a newonset weight-training program, but he was ultimately diagnosed with a germ cell tumor.

\section{CASE REPORT}

A 43-year-old male with no significant medical history presented with acute right groin and flank pain that woke him from sleep the night prior to admission. The patient described the pain as shooting, radiating to the right lower quadrant, and constant in nature. The patient was able to void and reported dark yellow urine. He denied any fever or chills. Upon further questioning, he described a new strength-training routine involving heavy back squatting exercises the 3 days prior to admission. At baseline, the patient was a physically fit individual who was active with his occupation as an ocean diver. He denied any use of illicit 

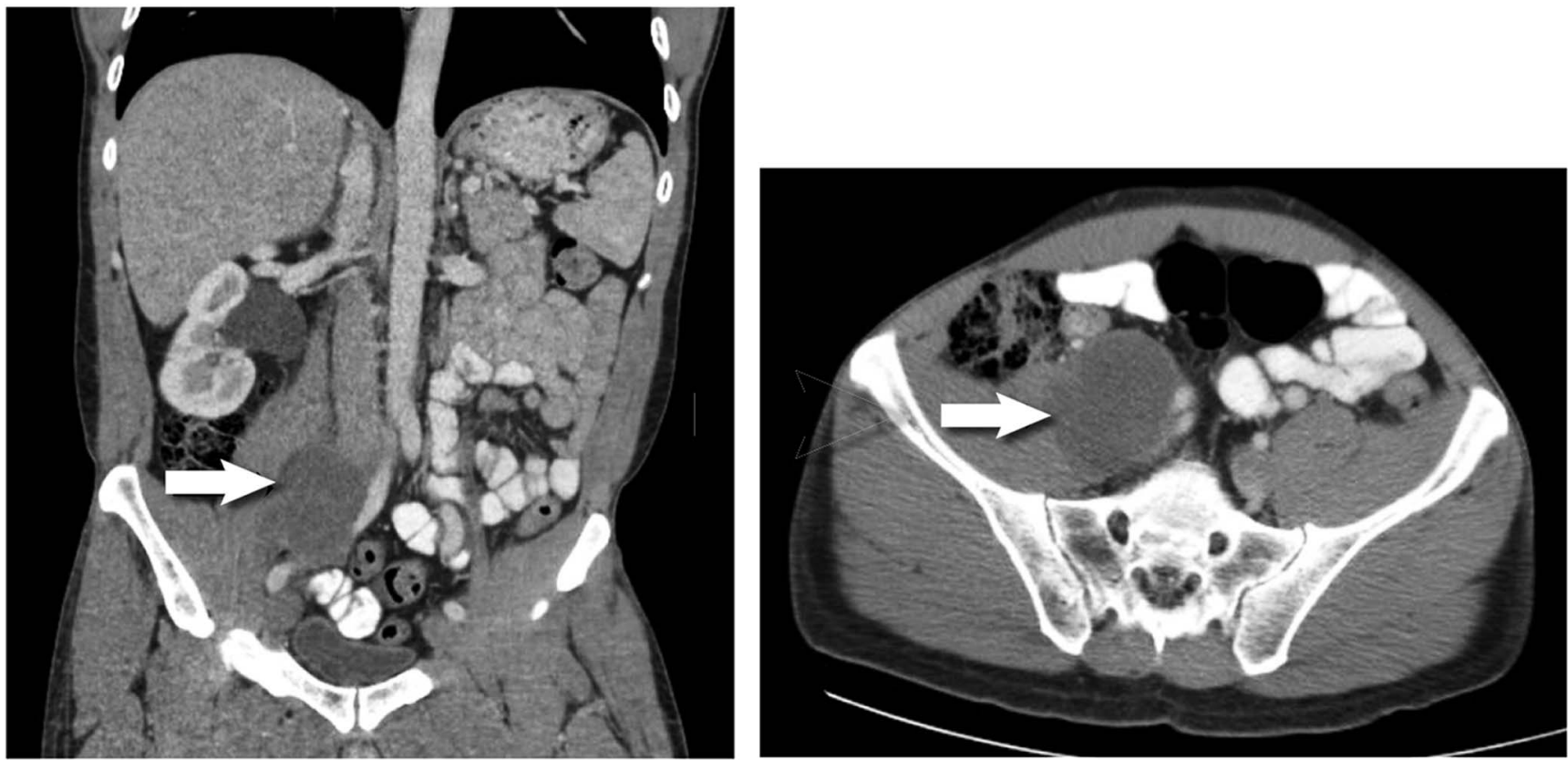

A

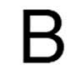

Figure 1. Computed tomography of abdomen and pelvis with intravenous contrast from initial hospital admission in (A) coronal view and $(B)$ axial view shows a $6.5 \times 5.3 \times 6.8-\mathrm{cm}$ right psoas hypoattenuating mass (arrow) compressing the right ureter with proximal ureteral and right kidney collection system dilatation. Medial deviation of the right iliac arterial vasculature and compression of the right venous iliac vasculature were present.

substances and intravenous (IV) drug use, and he had no history of human immunodeficiency virus (HIV).

Admission vitals were unremarkable; the patient was afebrile with a heart rate of $70 \mathrm{bpm}$. On physical examination, the patient had pertinent right groin pain radiating to the right lower quadrant and right testicular region. He had no loss of range of motion, full sensation, and full strength. Neurologic examination was unremarkable. Significant laboratory values included creatinine $0.8 \mathrm{mg} / \mathrm{dL}$ (reference range, 0.7-1.3 mg/dL), aspartate aminotransferase 74 units/L (reference range, 15-37 units/L), alanine aminotransferase 34 units/L (reference range, 14-59 units/L), and white blood cell count $10.5 \times 10^{3} / \mathrm{microL}$ (reference range, 4.3-10 $\times$ $\left.10^{3} / \mathrm{microL}\right)$ with $60 \%$ neutrophils and no bands. Urinalysis was unremarkable, with no blood or red blood cells. C-reactive protein was mildly elevated at $0.8 \mathrm{mg} / \mathrm{L}$ (reference range, $0.0-0.3 \mathrm{mg} / \mathrm{L})$.

Computed tomography (CT) of abdomen and pelvis with IV contrast showed a $6.5 \times 5.3 \times 6.8-\mathrm{cm}$ right psoas hypoattenuating mass compressing the right ureter with proximal ureteral and right kidney collection system dilatation. Medial deviation of the right iliac arterial vasculature and compression of the right venous iliac vasculature were present (Figure 1). CT imaging Hounsfield unit (HU) measurement of the psoas mass showed uptake of approximately 35-40 $\mathrm{HU}$, suggesting a pattern outside simple fluid uptake and close to the $\mathrm{HU}$ of blood. ${ }^{3}$ Core biopsies of the cystic mass revealed bloody purulent fluid and fragments of mature skeletal muscle. A 10-Fr drain was placed. Three days later, repeat CT of abdomen and pelvis with IV contrast to assess drainage demonstrated persistent hypoattenuating mass, although it was mildly reduced in size to $5.9 \times 4.7 \times 6.0 \mathrm{~cm}$. The mass still compressed the right ureter with unchanged proximal ureteral and right kidney collection system dilatation. Persistent medial deviation of the right iliac arterial vasculature and compression of the right venous iliac vasculature were noted (Figure 2). The patient was hemodynamically stable, able to void, and controlled on oral pain medications. The pigtail drain was upsized to 12-Fr and left in place, and the patient was discharged home with clinic follow-up.

Four weeks later, the patient returned to the emergency department with increasingly worse groin pain radiating down the right leg and accompanied by right inguinal edema. The patient also mentioned intermittent fevers and chills and urinary hesitancy. Repeat CT of abdomen and pelvis with IV contrast showed an interval increase in size of the heterogeneous hypoattenuating right psoas mass that now measured $8.1 \times 8.1 \times 9.1 \mathrm{~cm}$ in its greatest anteroposterior, transverse, and craniocaudal dimensions, respectively (an increase from $5.9 \times 4.7 \times 6.0 \mathrm{~cm}$ approximately 1 month prior). The pigtail drain was seen abutting the lateral anterior margin of the mass. Slightly increased right hydronephrosis and proximal hydroureter were noted secondary to increased mass effect. Repeat CT-guided right psoas mass biopsy was performed, accompanied by percutaneous drain removal. The patient wished to be discharged home on pain control and await biopsy results.

This time, pathology results noted "cohesive clusters of atypical cells," and the specimen was sent for expert further review. The histologic sections revealed a tumor with papillary and solid growth composed of cells featuring moderate pleomorphism. Antibodies against CD31, FLI-1, 


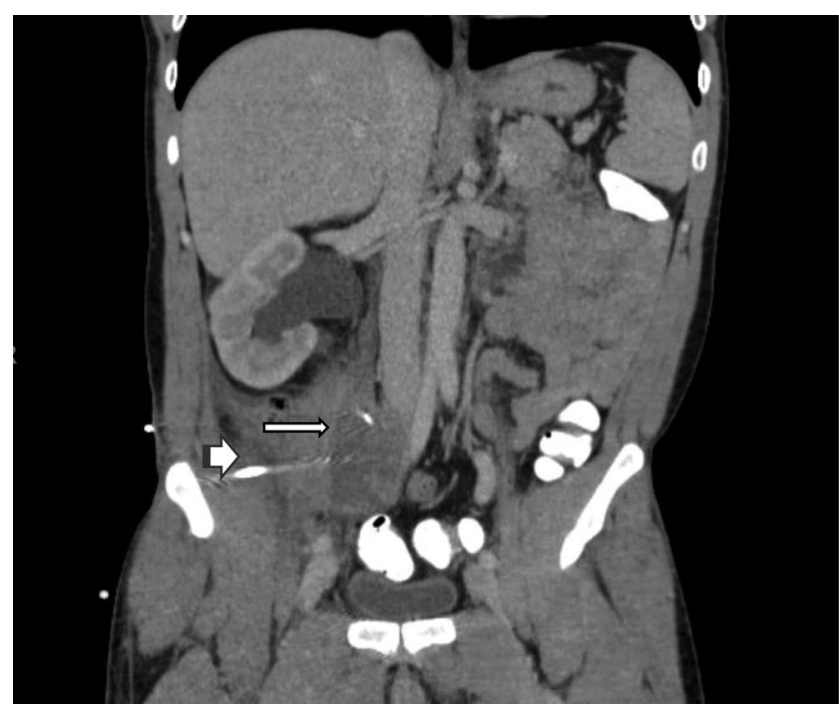

Figure 2. Computed tomography of abdomen and pelvis (coronal view) with intravenous contrast obtained 3 days after admission and initial psoas drain placement (arrowhead) shows persistent hypoattenuating mass, mildly reduced in size to $5.9 \times 4.7 \times 6.0 \mathrm{~cm}$ (arrow). The mass still compresses the right ureter, with unchanged proximal ureteral and right kidney collection system dilatation. Persistent medial deviation of the right iliac arterial vasculature and compression of the right venous iliac vasculature were noted.

keratin 34ßE12, SALL-4, OCT-4, and glypican-3 demonstrated diffuse and strong staining of the tumor cells with cytokeratin AE1/AE3, GATA3, SALL-4, glypican-3, and focal staining with alpha-fetoprotein and placental alkaline phosphatase. The review panel unanimously concluded that the morphology and immunohistochemical features supported a diagnosis of yolk sac tumor. The patient's serum alphafetoprotein level was $>5,000 \mathrm{ng} / \mathrm{mL}$ (reference range, 0.1-8.1 $\mathrm{ng} / \mathrm{mL}$ ), and serum beta-human chorionic gonadotropin was $<1 \mathrm{mlU} / \mathrm{mL}$ (reference range, $0-1 \mathrm{mlU} / \mathrm{mL}$ ). The patient was notified of his results. He complained of continual uncontrolled pain and intermittent fevers and was readmitted to the hospital a few weeks later. Testicular ultrasound showed only multiple punctate calcifications in the right testicle and no masses (Figure 3). Repeat CT of abdomen and pelvis with IV contrast demonstrated a further increase in the right psoas mass size to $8.9 \times 12.7 \times 10.0 \mathrm{~cm}$ in its greatest anteroposterior, transverse, and craniocaudal dimensions, respectively (Figure 4) compared to $8.1 \times 8.1 \times 9.1 \mathrm{~cm}$ at the patient's previous visit. CT demonstrated persistent left lateral displacement of the right common and external iliac arteries and the associated veins, along with increased right hydronephrosis and proximal hydroureter. Cardiovascular surgery noted no emergent vascular compromise on clinical examination and imaging. CT of the chest and magnetic resonance imaging of the brain as part of the malignancy workup were negative. Nuclear medicine bone scan showed only an old healing third rib nonpathologic fracture. An interventional radiology-guided ureteral metal stent was placed.

The patient's malignant neoplasm was germ cell in origin, either a metastatic focus from a testicular primary vs

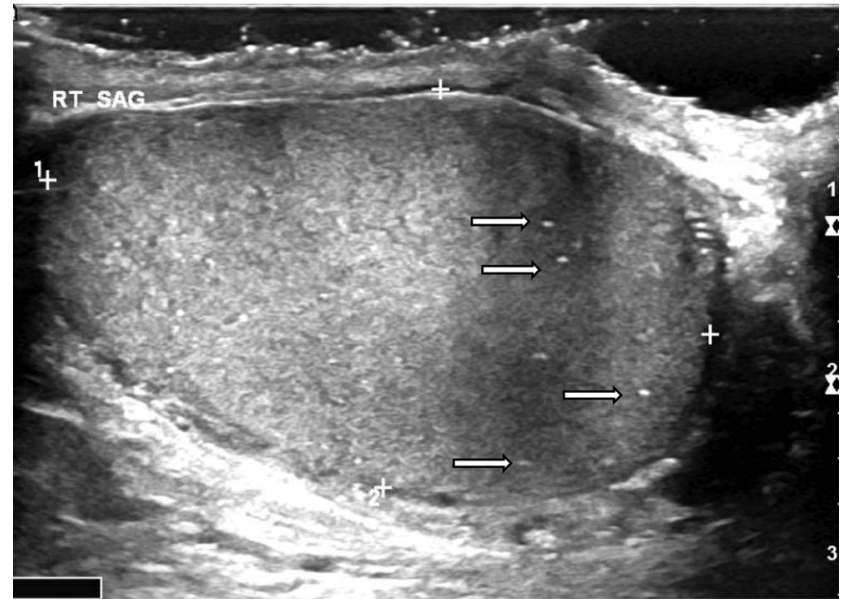

Figure 3. Scrotal ultrasound obtained after repeat pathology suggesting germ cell tumor shows multiple right punctate testicular microlithiasis and calcifications (arrows).

a primary extragonadal tumor. The alpha-fetoprotein level of $>5,000 \mathrm{ng} / \mathrm{mL}$ confirmed an intermediate risk. The consensus treatment for this T1N0M1 tumor invading the psoas wall was to administer 4 cycles (every 21 days) of BEP (bleomycin + etoposide + cisplatin) chemotherapy, surveillance of the alpha-fetoprotein during the cycle, and restaging at the end of 4 cycles with consideration of right orchiectomy \pm lymph node dissection, depending upon the patient's response and presence of residual disease. Full prechemotherapy tests, including pulmonary function testing, were performed. At the time this case was written, the patient had started cycle 1 of BEP and had developed a right lower extremity deep venous thrombosis pretreatment, a few days prior to chest

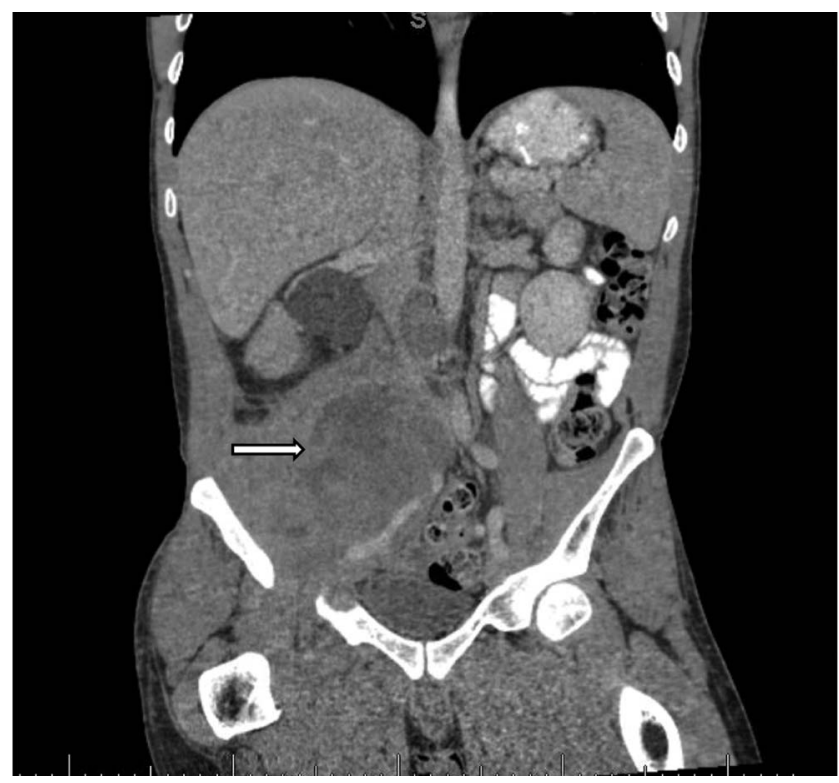

Figure 4. Computed tomography of abdomen and pelvis (coronal view) with intravenous contrast obtained after repeat pathology suggesting germ cell tumor and closest to chemotherapy initiation shows interval-enlarged hypoattenuating mass measuring $8.9 \times 12.7 \times 10.0 \mathrm{~cm}$ (arrow). 
port placement. Lovenox (enoxaparin sodium) therapy was initiated, and the patient had full oncologic and medical follow-up.

\section{DISCUSSION}

The proper differentiation between a burned-out germ cell tumor originating from testicular focus vs a primary retroperitoneal germ cell tumor must be made to guide the treatment course. Testicular ultrasound did not show any masses or evidence of cancer in our patient. However, ultrasound did display testicular microlithiasis or punctate microcalcifications. A relationship between testicular microlithiasis and testicular cancer has been shown; however, the majority of occurrences are found in noncancer patients. ${ }^{4}$ The grade or amount of testicular microlithiasis does not correlate with cancer risk. ${ }^{4}$ Numerous studies have also investigated the necessity of ipsilateral orchiectomy in the presence of extragonadal germ cell tumor and negative ultrasound primary tumor findings. ${ }^{5}$ Although studies have been inconsistent, ipsilateral orchiectomy does not appear to be necessary if a normal testicle is found on the ultrasound. ${ }^{5}$ Our patient's response to treatment will help to delineate the origin of his iliopsoas germ cell tumor. If the tumor is completely extragonadal in origin, the patient should have a good response to the chemotherapy course. If not, suspicion of at least a histologic presence of testicular cancer should remain, and consideration of right orchiectomy \pm lymph node dissection is warranted.

The literature supports 2 theories for the plausible origin of a primary extragonadal germ cell tumor. The first supports origination from germ cells that migrated from the yolk sac to contribute to genital ridge formation but remained trapped in other tissues. ${ }^{6}$ The second theory suggests that reverse migration of transformed gonadal cells to other locations may contribute. ${ }^{7}$

Upon our patient's initial presentation, the presence of a tumor was not the leading differential diagnosis. Anatomically, tumor invasion can have direct and random sequestration of retroperitoneal fascial planes, in contrast to infectious or inflammatory processes. The presence of fascial plane discontinuity, bone destruction, and profound lymphadenopathy all point toward tumor involvementcharacteristics not present radiographically in our case. ${ }^{8}$ Initially, we used a thorough understanding of muscle biomechanics and radiography to help delineate the most likely etiology of the patient's traumatic psoas hemorrhagic lesion-back squats. The patient had recently engaged in a strenuous back squat regimen with a personal trainer that had involved a heavy barbell on the patient's shoulder and squatting to a 90-degree angle perpendicular to ground level with the feet shoulder width apart. To determine the most plausible explanation for the patient's psoas mass, we focused on the biomechanics of the squat, delving into the anatomy and mechanistic properties of the involved muscles, as the anatomic explanation seemed the most logical. The iliopsoas helps maintain an erect posture and, most important, aids in hip flexion. The most plausible explanation at the time was vigorous and repetitive squats, an exercise naïve to the patient, that caused prolonged irritation or sporadic trauma to the psoas during the eccentric (lowering) phase of the squat.
In addition to a focused history and clinical examination and an understanding of muscle biomechanics, delineating a traumatic hematoma from other iliopsoas compartment lesions is vital. Primary iliopsoas infection is rare, but the bowel (Crohn disease, appendicitis, diverticulitis), kidneys (perinephric abscesses), and bones are potential sources of secondary infection. ${ }^{1}$ Pyogenic abscesses typically present as lesions of low attenuation on CT with marginal enhancement after contrast injection, with more than $50 \%$ of cases having a gas pattern visualized. ${ }^{1,2,9}$ Although our patient presented with hydronephrosis and hydroureter, he displayed no radiographic evidence of abscess, and his urinalysis and culture were unremarkable. Our patient also did not have HIV or tuberculosis, known culprits of such radiographic pyogenic findings. , $^{1,9}$ A proper working differential diagnosis helped prevent unnecessary antibiotic use in our case.

Iliopsoas hematomas may be accompanied by wellrecognized risk factors such as chronic anticoagulation, trauma, or recent intervention. Other cases, such as ours, require a more thorough anatomic understanding and patient history. On CT, acute hematomas or hemorrhages present with hyperdense contents $(H U>30)$, differentiating hematomas or hemorrhages from simple fluid or adjacent organ or structure HU uptake pattern. ${ }^{3}$ The region of interest remains unenhanced after contrast injection, as in our case, except in cases of active bleeding. ${ }^{1,2}$ Chronic hematomas, however, are oftentimes indistinguishable from necrotic tumors or abscesses. Our understanding of the radiographic uptake pattern, along with aspirate pathology confirming blood and skeletal muscle, appeared to confirm our initial suspicion of an iliopsoas hematoma as the iliopsoas compartment lesion and the culprit of the patient's presenting symptoms. However, the hypothesis was later debunked with the patient's continual and worsening symptoms, radiographic deterioration, and repeat iliopsoas mass biopsy.

Regarding the necessity of drainage and aspirate for diagnosis confirmation, the iliopsoas hematoma was compressing the adjacent vasculature and causing a nonobstructive hydroureter and hydronephrosis. Drainage decompression and imaging reanalysis 3 days later after initial presentation were prudent to assess adjacent organ function. We felt aspirate and pathologic analysis was warranted to fully classify the lesion, as radiographic intervention was already in place to prevent nearby ureter and organ obstruction, and no further harm would be done to complete the workup. Clinical persistence with repeat psoas mass biopsy and pathologic expertise was required to yield the correct diagnosis.

\section{CONCLUSION}

Biomechanical understanding of injury pattern combined with prudent radiographic knowledge can synergistically help to delineate iliopsoas compartment lesions in a timely and accurate manner. When clinical symptoms persist or worsen, revisiting the initial diagnosis and considering adjunct objective diagnostic workup are imperative.

\section{ACKNOWLEDGMENTS}

The authors have no financial or proprietary interest in the subject matter of this article. 


\section{REFERENCES}

1. Muttarak M, Peh WC. CT of unusual iliopsoas compartment lesions. RadioGraphics. 2000 Oct;20 Spec No:S53-S66. doi: 10.1148/radiographics.20.suppl_1.g00oc07s53.

2. Torres GM, Cernigliaro JG, Abbitt PL, et al. Iliopsoas compartment: normal anatomy and pathologic processes. RadioGraphics. 1995 Nov;15(6):1285-1297. doi: 10.1148/radiographics.15.6.8577956.

3. Zech WD, Jackowski C, Buetikofer Y, Kara L. Characterization and differentiation of body fluids, putrefaction fluid, and blood using Hounsfield unit in postmortem CT. Int J Legal Med. 2014 Sep;128(5):795-802. doi: 10.1007/s00414-014-1030-0.

4. Yee WS, Kim YS, Kim SJ, Choi JB, Kim SI, Ahn HS. Testicular microlithiasis: prevalence and clinical significance in a population referred for scrotal ultrasonography. Korean J Urol. 2011 Mar;52(3):172-177. doi: 10.4111/kju.2011.52.3.172.
5. Gîngu CV, Mihai M, Baston C, et al. Primary retroperitoneal seminoma - embryology, histopathology and treatment particularities. Rom J Morphol Embryol. 2016;57(3):1045-1050.

6. Lane RH, Stephens DH, Reiman HM. Primary retroperitoneal neoplasms: CT findings in 90 cases with clinical and pathologic correlation. AJR Am J Roentgenol. 1989 Jan;152(1):83-89. doi: 10.2214/ajr.152.1.83.

7. Egund N, Ekelund L, Sako M, Persson B. CT of soft-tissue tumors. AJR Am J Roentgenol. 1981 Oct;137(4):725-729. doi: 10.2214/ajr.137.4.725.

8. Feldberg MA, Koehler PR, van Waes PF. Psoas compartment disease studied by computed tomography. Analysis of 50 cases and subject review. Radiology. 1983 Aug;148(2):505-512. doi: 10.1148/radiology.148.2.6867350.

9. Lenchik L, Dovgan DJ, Kier R. CT of the iliopsoas compartment: value in differentiating tumor, abscess, and hematoma. AJR Am J Roentgenol. 1994 Jan;162(1):83-86. doi: 10.2214/ajr.162.1.8273696.

This article meets the Accreditation Council for Graduate Medical Education and the American Board of Medical Specialties Maintenance of Certification competencies for Patient Care and Medical Knowledge. 